\title{
Pericardial Effusion - Melioidosis or Tuberculosis
}

\author{
SMITHA BHAT, ${ }^{1}$ JAYAPRAKASH ALVA, ${ }^{2}$ MOHAMMAD ASHRAF, ${ }^{3}$ SOURAB HIREMATH, ${ }^{4}$ SHISHIR DUBLE $^{5}$
}

\begin{abstract}
:
Melioidosis which is endemic in South East Asia and Northern Australia is now being reported from the Indian subcontinents as well. Meliodosis rarely presents as pericardial effusion. We report a case of B. pseudomallei causing pericardial effusion from India, where the most common infective cause of pericardial effusion is Mycobacterium tuberculosis. The chronic presentation of melioidosis is not unlike tuberculosis, and the clinician must have a high index of suspicion to diagnose meliodosis, especially in geographical areas where both tuberculosis and melioidosis are prevalent.
\end{abstract}

Keyword: Pericardial Effusion, Melioidosis

\section{Introduction:}

Melioidosis is widespread in Thailand, Australia, Singapore and Malaysia. there are indications that infection with Burkholderia pseudomallei may be more common than previously recognised. ${ }^{1}$ It's presence in India is gradually being recognised. Melioidosis can involve almost any organ system; however, pericardial involvement is rare. We present a case of Burkholderia pericardial effusion in a male who presented with fever and breathlessness.

\section{Case Report:}

The patient, a 36 year old construction worker from Mangalore, which is a town on the western coast of Karnataka, a state in south India, presented to hospital with a history of intermittent fever associated with chills for 21 days. He gave a history of pedal edema for 3 days, and worsening breathlessness and decreased urine output from the day preceding admission. He also complained of hiccups. There was no history of cough, body ache, burning micturition or loose stools. There was no history of chest pain or wheeze.

On examination, he was breathless at rest in the orthopneic position. His pulse rate was 110 / minute with no paradoxus, his blood pressure in the right arm supine position was 100/70 $\mathrm{mm} \mathrm{Hg}$ and his jugular venous pressure was elevated. Lungs were clear and there was tender hepatomegaly $5 \mathrm{~cm}$ below the right costal margin. Auscultation of the cardiovascular system was unremarkable except for tachycardia.

1. Consultant Physician and Associate Professor of Medicine. Convener, MEU, Father Muller Medical College, Mangalore; Phone - (M) - 9845162167, (O) - 08242238271

2. Father Muller Medical College, Mangalore

Correspondence : Dr. Jayaprakash Alva; E-mail: japal6@ rediffmail.com
Labs revealed neutrophilic leucocytosis, an ESR of 55 $\mathrm{mm}$ in the first hour, and normal renal and liver function tests.

Sonography showed congestive Hepatomegaly and ascites. Chest $\mathrm{x}$ ray showed cardiomegaly with water bottle appearance. An urgent echocardiogram was done which revealed a large pericardial effusion with tamponade features. An emergency pericardiocentesis was done and a pigtail catheter left in place. The patient improved symptomatically after pericardiocentesis, and $700 \mathrm{ml}$ of straw coloured fluid was drained and sent for biochemical analysis, gram stain and culture sensitivity. Fluid protein and ADA was elevated. Gram stain showed numerous pus cells and occasional mononuclear cells. AFB staining did not show acid fast bacilli. The blood culture isolated no bacteria.

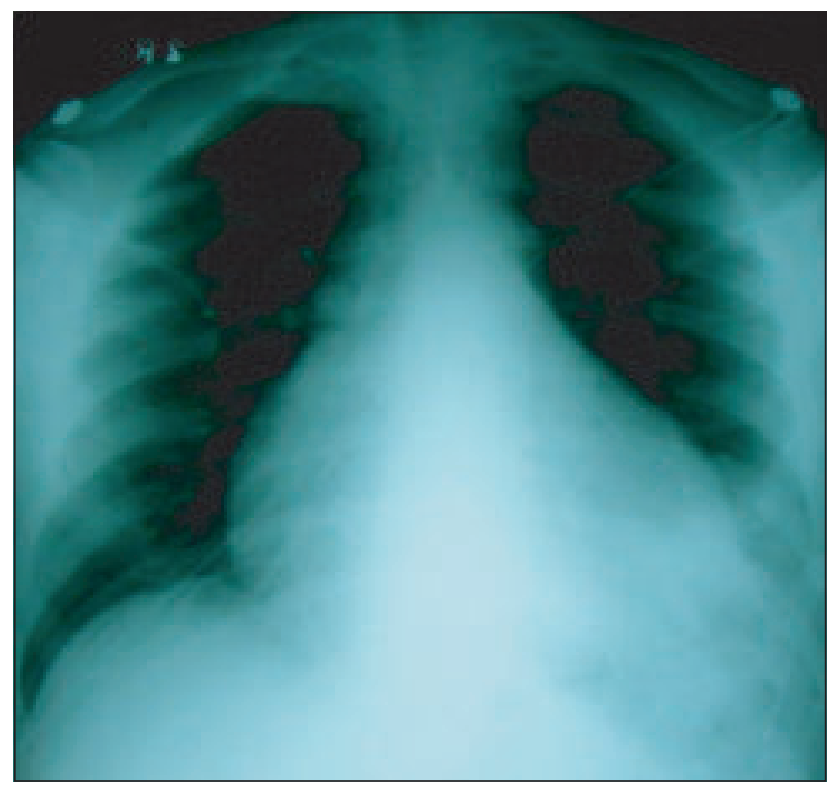

Fig.-1: Chest x ray 
After 4 days we received the reports of the pericardial fluid culture which grew Burkholderia pseudomallei.

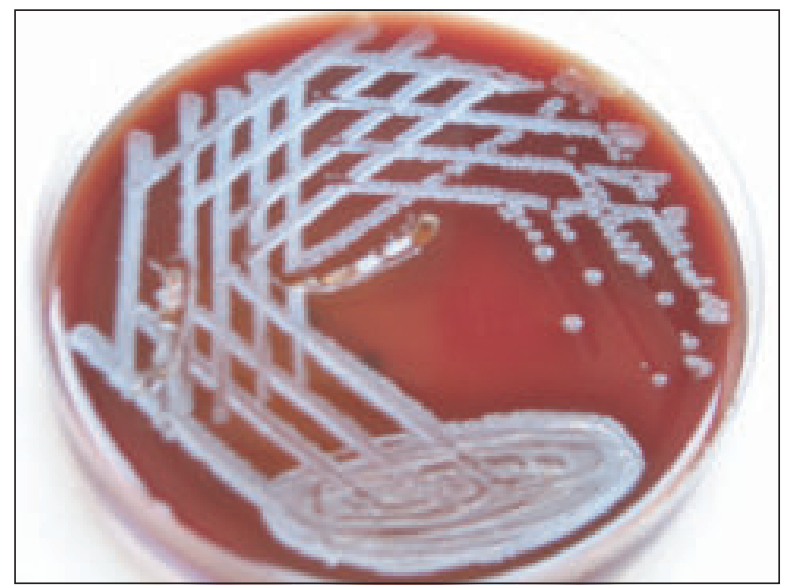

Fig.-2: B. pseudomallei growing on blood agar

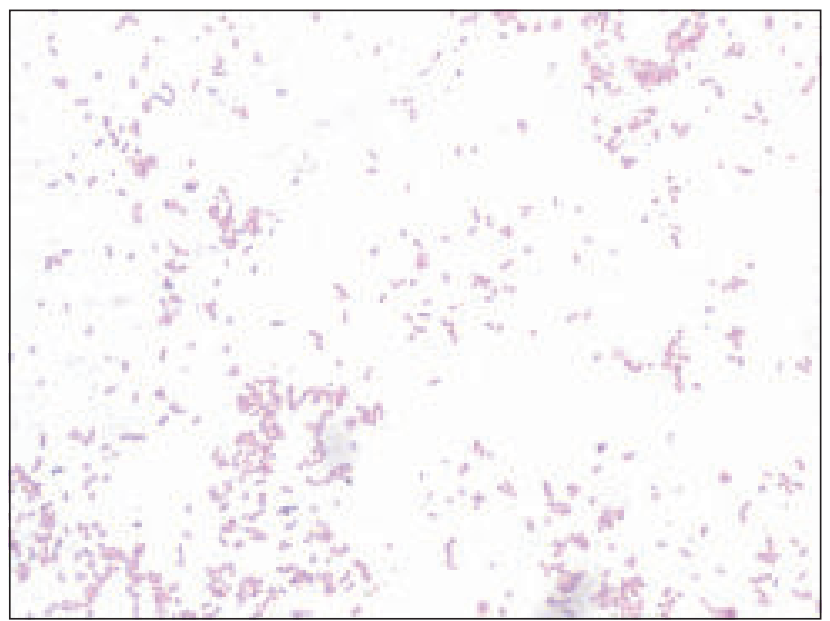

Fig.-3: Gram stain of B, pseudomallei.

The patient was started on ceftazidime and became afebrile $48 \mathrm{hrs}$ after the first dose of ceftazidime. After completing a 2 week course of intravenous ceftazidime qid in hospital, the patient was discharged. Tablet Doxycycline $100 \mathrm{mg}$ bd and tablet cotrimoxazole double strength bd and folic acid were continued in the eradication phase for 3 months.

\section{Discussion:}

Melioidosis, also known as Whitmore's disease is caused by Burkholderia pseudomallei. It is endemic in Southeast Asia and Northern Australia. However, there are indications that the epidemiology of melioidosis is changing, with cases and case clusters being recognized outside these areas as well. Melioidosis is being increasingly diagnosed in India. ${ }^{2}$

B. pseudomallei is a free living motile gram negative bacillus normally found in soil, ponds and rice fields. It is a facultative intracellular organism, and its replication within polymorphonuclear leucocytes may be assisted by its polysaccharide capsule. ${ }^{3}$ It grows on most agar media. Infection can be acquired by inhalation, inoculation, and through exposure of contaminate water to abraded skin. The incubation period varies from 1 day to many years but is on an average $2-4$ weeks after exposure. ${ }^{4}$

Risk factors for melioidosis include alcoholism, diabetes, renal failure, cirrhosis, thalassemia and immunosuppresion. ${ }^{1,5}$ Our patient had none of these risk factors. The absence of risk factors does not eliminate the possibility of melioidosis - other studies have shown that up to $16 \%$ of patients with melioidosis have no identifiable risk factors. ${ }^{5}$

Melioidosis is a great mimicker and has a number of presentations varying from clinically inapparent infections to acute septicaemia. The infection may be acute, subacute or chronic. Burkholderia also has the ability to cause latent infections, and therefore may occur in travellers many days after they leave endemic areas.

Pulmonary infections, either acute or chronic, are the most common manifestation of melioidosis and present acute infections have presentations ranging from acute bronchitis to extensive necrotizing pneumonia. In some areas, melioidosis is a common cause of fatal community acquired pneumonia. ${ }^{6}$ Pneumonia due to Burkholderia is characterized by fever with rigors, productive cough and marked tachypnea. Chronic pulmonary infections present like tuberculosis with low grade fever, night sweats and productive cough, and chest $\mathrm{x}$ ray showing upper lobe infiltrates with thin walled cavities. ${ }^{3}$

Melioidosis is a disease distinguished by its tendency to abscess formation and septicaemia. B. pseudomallei commonly presents as septicaemia and multiple abscesses with fever, headache, abdominal pain disorientation, muscle tenderness and joint pain. ${ }^{4}$ Other clinical features include hepatosplenomegaly, osteomyelitis and pleural effusion.$^{7}$ It may also present with skin ulcers or abscesses with nodular lymphangitis and lymphadenitis.

Pericardial effusion due to Burkholderia pseudomallei is uncommon. ${ }^{8}$ Tuberculosis is an important infectious cause of pericardial effusion in India, and many patients with pericardial effusion are empirically started on ATT. Burkholderia pericardial effusion also has a subacute to chronic course, and may be mistaken for tuberculous pericarditis. Patients who have been empirically started on antituberculous therapy (ATT) have been subsequently proven to have Melioidosis. ${ }^{9}$ Apart from culture of pericardial fluid, there is no infallible method to distinguish 
between tuberculous and Burkholderia pericardial effusion. Our case had neutrophilic leucocytosis, and a previous study too showed that neutrophilic leucocytosis was comparatively more frequent in effusion due to B. pseudomallei, though this difference was not statistically significant. ${ }^{10}$

The diagnosis of melioidosis requires a high index of suspicion. It must be considered in a patient from an endemic area who presents with fever, lower respiratory infection or a chest $\mathrm{x}$-ray suggestive of tuberculosis in the absence of tuberculosis bacilli in the sputum. Gram staining of exudates or effusion reveals small bipolar irregularly staining gram negative rods with safety pin appearance. Culture of blood/ fluid on agar media shows the typical colonies of B. pseudomallei.

The treatment of melioidosis involves therapy with appropriate antibiotics and drainage of exudates/effusions. The antibiotic of choice is ceftazidime or imipenem for $10-$ 30 days. This is followed by eradication therapy with a combination of doxycycline and cotrimoxazole for $12-20$ weeks.

\section{Conclusion:}

The epidemiology and clinical features of melioidosis are changing - it is affecting more places, affecting patients who do not have the recognised risk factors, and presenting in more clinical ways. Its subacute and chronic presentation is similar to tuberculosis and it occurs in areas where tuberculosis is also a common public health problem. It is essential for the clinician to keep melioidosis in the list of differential diagnoses for a variety of presentations and to order appropriate microbiological investigations. Melioidosis can be fatal if untreated and tends to relapse if only partially treated. Patient education and motivation to complete the course of antibiotics is paramount.

\section{Conflict of Interest : None}

\section{References:}

1. DA, Dance Melioidosis as an emerging global problem. 2000, Acta Trop

2. K Vidyalakshmi, B Shrikala, B Bharathi and U Suchitra. Melioidosis: an under-diagnosed entity in western coastal India: a clinico-microbiological analysis. Indian J Med 2007, Microbiol
3. Ramphal, Reuben. Infections Due to Pseudomonas Species and Related Organisms. [book auth.] Fauci. Kasper, Hauser, Jameson,Loscalzo Longo. Harrison's Principles of Internal Medicine. 2011

4. http://www.cdc.gov/melioidosis/health-care-workers/ index.html. www.cdc.gov. [Online] May 23, 2011. [Cited: December 30, 2011.]

5. Saravu, K, et al Melioidosis in Southern India: Epidemiological and clinical profile., Southeast Asian Journal of Tropical Medicine and Public Health, 2010;Vol. 41

6. Currie BJ, Fisher DA, Howard DM, Burrow JN, Selvanayagam S, Snelling PL, Anstey NM, Mayo MJ The epidemiology of melioidosis in Australia and Papua New Guinea. Acta Trop.2000; 74

7. Saravu, K; Mukhopadhyay, C; Vishwanath, S; Valsalan, R; Docherla, M Melioidosis in Southern India: Epidemiological and clinical profile. Southeast Asian Journal of Tropical Medicine and Public Health, 2010; 41:2

8. Endemic melioidosis in tropical northern Australia: a 10year prospective study and review of the literature. Clinical Infectious Diseases. 2000

9. Vidyalakshmi K, Chakrapani M, Shrikala B, Damodar S, Lipika S, Vishal Tuberculosis mimicked by melioidosis. Int J Tuberc Lung Dis, 200812;S. 10

10. Songsak Kiatchoosakun, Churairat Kularbkaew, Ploenchan Chetchotisakd Melioidosis Mimicking Tuberculous Pericarditis. Clinical Infectious Diseases 2010, 51

11. Chaowagul W, Chierakul W, Simpson AJ, Short JM, Stepniewska K et al. Open-label randomized trial of oral trimethoprim-sulfamethoxazole, doxycycline, and chloramphenicol compared with trimethoprimsulfamethoxazole and doxycycline for maintenance therapy of melioidosis. Antimicrob Agents Chemother 2005

12. Mukhopadhyay C, Chawla K, Krishna S, Nagalakshmi N, Rao SP, Bairy I Emergence of Burkholderia pseudomallei and pandrug-resistant non-fermenters from southern Karnataka, India. Trans R Soc Trop Med Hyg.2008.

13. Melioidosis: epidemiology, pathophysiology, and management. Currie, AC Cheng and BJ. 18, 2005, Clin Microbiol Rev

14. G Borgherini, P Poubeau, F Paganin, S Picot, A Michault and F Thibault Melioidosis: an imported case from Madagascar. J Travel Med.2006, 13 